\title{
The influence of oil-refrigerant mixture on thermal and physical processes on friction joints
}

\author{
Elena Grigorieva ${ }^{1 *}$, Sergei Kulichkov ${ }^{2}$, and Larisa Andreeva ${ }^{3}$ \\ ${ }^{1}$ Far Eastern State Technical Fisheries University, Engineering Disciplines Department, 52 B Lugovaya \\ Str., Vladivostok 690087, Russia \\ ${ }^{2}$ Far Eastern State Technical Fisheries University, Engineering Disciplines Department, 52 B Lugovaya \\ Str., Vladivostok 690087, Russia \\ ${ }^{3}$ Far Eastern Federal University, Polytechnic Institute, Engineering Department, Campus 10 Ajax \\ Bay, Russky Island, Vladivostok 690922, Russia
}

\begin{abstract}
The paper deals with the influence of refrigerants dissolved in oil on the thermo-physical properties of the lubricant and the operation of friction joints in refrigeration machines. It is demonstrated that in the axial and circumferential directions the bearing temperature, if lubricated with a mixture of T-30 - R-22 is significantly lower than when lubricated with T30 oil.
\end{abstract}

\section{Introduction}

Strict requirements are imposed to performance characteristics of oils applied in refrigeration equipment.

Oils should have stability, antioxidant resistance, anticorrosion properties, mobility at low temperatures, lubricating efficiency. The properties specified determine reliability and feasibility of oils application in conditions of refrigeration units operation.

The main characteristic of lubricating agents is viscosity, which depends significantly on temperature. Moreover, the change in oil viscosity is described by exponential and power characteristics.

\section{Literature overview}

Kasper Górny et al. (2016) offered the procedure for estimation of lubricating properties of oils for refrigeration compressors in combination with refrigerant. Lubricating properties of mixtures consisting of polyether oils and refrigerant R134a and mineral oils and refrigerant R290 were estimated. It was found out that wear of samples after testing in oil-refrigerant mixture is significantly higher than in oil without a refrigerant [1].

The studies of Wang Chuang et al. (2018) proved that small amount of refrigerant dissolved in oil promotes stability of rotor bearing system with small clearance radius and elongation in twin screw refrigeration compressor [2].

* Corresponding author: gev1320010@mail.ru 
Wu Jianhua et al. (2018) determined the temperature of saturated solution of the oil pan mixture. The influence of R290 solubility and mixture viscosity in oil pan was studied. It was found out that viscosity of R290/NM100 MO mixture is enough for lubrication of thrust bearings [3].

Yanjun Sun et al. (2020) measured dynamic viscosity of polyether oils and refrigerants R1234yf and R1234ze. The results showed that mixture viscosity dramatically dropped with the increase of molar fraction of refrigerants in oil, especially at lower temperatures [4].

M. Wasim Akram et al. (2014) carried out a comparative study of tribological properties of different lubricants in the medium of HFO-1234yf refrigerant, the influence of mutual solubility of oils and refrigerants for tribo-formation. The studies showed better tribological characteristics of the polyalkyleneglycol / HFO-1234yf system compared to the polyether oil / HFO-1234yf system. Moreover, in the first case adhesive wear caused by plasticity was found, and in the second case - abrasive wear caused by micropitting [5].

M. Wasim Akram et al. (2013) carried out a comparative tribological study under conditions of inadequate lubrication with the use of HFO-1234 yf and R134a refrigerants and the gray cast-iron interface. Loading and wearing experiments showed that HFO-1234 yf refrigerant is better than $\mathrm{R} 134 \mathrm{a}$ refrigerant [6].

Rui Zhai et al. (2019) gave measurement results of miscibility of refrigerants (R1234ze (E), RE170, R22, R142b, R152a, R161 and R227ea) with three mineral oils for the temperatures from $-70^{\circ} \mathrm{C}$ to $40^{\circ} \mathrm{C}$. It was found out that hydrochlorofluorocarbons, RE170 can be easily mixed with mineral oils [7].

Mitsuhiro Fukuta et al. (2020) addressed the problems of wetting properties of oilrefrigerant mixtures. Measurements of contact angle and interface tension of oil-refrigerant mixture showed that contact angle of polyalkylene glycol and $\mathrm{CO} 2$ decreased with the increase of refrigerant concentration [8].

Mathieu Garcia et al. (2013) studied three-dimensional behavior of viscosity and temperature in cross-section of a film in order to correspond viscosity and in axial direction in order to take into account potential temperature gradient that significantly changes bearing temperature profile [9].

Bachir Bouchehita et al. (2016) showed that non-linear phenomena in bearings are connected with the influence of temperature and deformation of lubricating film. Temperature has a greater influence on bearing characteristics than deformation [10]. [11].

BinYang et al. (2013) presented the lubricating model of piston-type compressor bearings

Yajing Lia et al. (2017) carried out experiments and computing simulation in order to study thermal and hydrodynamic characteristics of turbocharger rotor bearing system. Their work showed that the traditional two-dimensional model of the oil film will fail to predict oil film behavior due to the presence of undeveloped thermal boundary layers [12].

\section{Materials and methods}

Pressure has little effect on clean oil viscosity, but in cases of high pressure values, the dependence is determined by Barus formula.

Turbine oils T-22, T-30, T-46 are applied in steam and gas turbines, air and refrigeration centrifugal compressors (CC).

Refrigeration CC have full-pressure lubrication systems and the turbine oil used comes in contact with the refrigerant, which vapors fill up the upper part of the oil tank and the bearing chamber. At the same time, the vapor pressure is determined by the operation mode of the refrigeration plant, which includes CC. During operation and stand-by mode of the turbine set oil absorbs refrigerant vapors accompanied by the change in its viscosity. 
Viscosity value is determined not only by oil temperature, but the concentration of the dissolved refrigerant.

Refrigerant concentration in oil depends on temperature and pressure in the oil tank. The techniques used in laboratories for measuring viscosity of oil-refrigerant mixtures are based upon simple and idealized phenomena, while experiments are carried out under conditions that are remote from reality and usually don't reproduce real operation conditions of lubrication system of the centrifugal compressor. Therefore, in order to obtain data on the viscosity coefficients of mixtures of turbine oils with refrigerants prevailing in the lubrication systems of refrigerating $\mathrm{CC}$, a special viscometer was developed. It allows measuring viscosity of such mixtures as freon and oil that are close to the operating conditions of a turbocharger oil system.

Automatic viscometer is shown in fig. 1, and the wiring diagram is shown in fig. 3 .

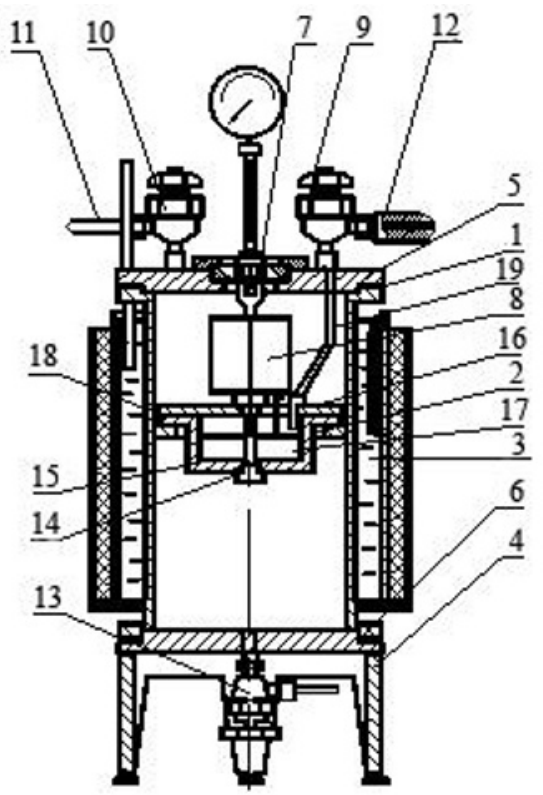

Fig. 1. Automatic viscometer.

The device consists of a pressure-tight chamber 1 with shut-off valves and pipelines and a reference pressure gauge on the cover of the chamber, an internal tank 2 with a shut-off valve and a bath 3 , which is a thermostat to maintain the required temperature, and a stand 4 with adjustable screws for device installation. Chamber 1 is made of steel grade 45 in the form of a cylinder. On the top cover 5 a panel 7 is fixed, where electrical contacts of the control circuit of the electromagnetic coil 8 , shut-off angle valves 9,10 with pipelines 11,12 are located. The bottom cover has a valve 13, which allows the oil-refrigerant mixture to be drained from the viscometer. The tank for the test mixture is a cylindrical steel vessel with a bottom in the form of a spherical segment. A sized drainage pipe with the inner diameter of $2.8 \mathrm{~mm}$ is fixed at the bottom of the tank. The pipe is closed with the stem 15 being the pin of an electromangnetic coil 8 fixed on the cover 16 of the internal tank. A copper-constantan thermocouple is mounted on the cover for measuring the temperature of the test mixture under and a pipe 19 for filling the internal tank with liquid. There is a guide installed in the tank. A float with electrical contacts made in the form of a disk and intended to register the rate of outflow of the fixed volume of mixture through the calibrated hole, moves along the guide. 
The control and measuring equipment includes a potentiometer Ш-63, a signal lamp for registering the rate of liquid outflow and a reference pressure gauge (accuracy class 0.04).

Preliminary the constant (water number) of the device was determined. For this purpose the cover with the electromagnetic coil, the shut-off valve and the thermocouple is removed from the inner tank and inserted into the thermostat. A clean and dry pin is inserted in the top hole of the drainage pipe. The inner surface of the tank is flushed with ethyl ether, distilled water and dried with air. Filtered distilled water at a temperature of $20^{\circ} \mathrm{C}$ is poured from the measuring flask to the top edge of the tank. A constant-temperature bath is fill up with the water of the same temperature. Emptied flask is placed under the tank drainage pipe, the pin is raised and all water is drained from the tank into the flask. After lowering the end into the drainage pipe, water from the flask is again poured into the tank. Water in the tank and the bath is carefully mixed with the help of a mixer. Having established by the thermometer that the water temperature in both reservoirs is $20^{\circ} \mathrm{C}$, and that within 300 seconds the temperature deviation does not exceed $0.2{ }^{\circ} \mathrm{C}$, the pin is raised, simultaneously running the stopwatch and observing the outflow of water from the tank, fixing the moment when the meniscus bottom edge reaches the circular mark on the flask corresponding to a $200 \mathrm{ml}$ capacity. The stopwatch will be stopped at that moment. The distilled water outflow is observed during 4 consecutive times. In our case the water number comprised $\tau_{20}^{\mathrm{H}_{2} \mathrm{O}}=57,3 \mathrm{~s}$.

Before each series of viscosity tests, tank 2 and its drainage pipe will be thoroughly washed with clean filtered light gasoline and dried with air. The cover with an electromagnetic coil, a stem, a thermocouple and a float with electrical contacts is installed on the top of the tank. At the same time a shut-off valve is operated to shut-off the drainage pipe; while the float with electrical contacts is places on the bottom of the tank. The assembled structure is placed into the chamber, on which the cover is installed and fixed with bolts.

To measure viscosity of oil-refrigerant mixture in the device, compressor crankcase and oil tank of a centrifugal machine, it is necessary to connect a previously vacuumed device to the oil chamber by pipeline 5 , and to the steam chamber by pipeline 6 . The device will be located so that its middle part is lower than the level of the mixture in the sampling point. Having determined the temperature of the test mixture and the pressure in the vapor chamber, identical conditions will be created in the viscometer by heating or cooling the thermostatic liquid 7 and pressure balancing by opening valve 8 . Through valve 9 the mixture from the sampling point by gravity flows through the pipeline 10 into the internal tank 2 (the supply under a slight excess pressure is possible). As the tank is filled with the mixture, the float with electrical contacts floats up and opens the electrical circuit, fixing the beginning of filling the tank with liquid. When the tank is filled with $200 \mathrm{ml}$ of oil-refrigerant mixture, the float closes the circuit, signaling this fact. The supply of the mixture stops.

The temperature of the incoming liquid is recorded with the help of the thermocouple. Once the preset temperature has been established, hold for $120-300$ seconds and simultaneously turn on the stopwatch and the electromagnetic coil. Upon expiration of the fixed volume flow, the stopwatch stops, the coil turns off. The enlarger viscosity is determined by the equation:

$$
A V_{t}=\frac{\tau_{t}}{\tau_{20}^{H_{2} O}}
$$

where: $\tau_{t}$ - the flow time of $200 \mathrm{ml}$ of mixture from the viscometer at the test temperature.

The oil tank of the previously vacuumed system is filled with the test turbine oil. Electric heater 11 brings the temperature of the lubricant to the specified temperature for this experiment. Temperature control is performed by six thermocouples located at different depths of the oil tank, selected through a multi-point switch to a potentiometer. Oil is circulated through filters and the heat exchanger with the help of a pump. Maintaining a constant temperature of the oil circulating in the system is achieved by means of electric heaters that are periodically switched on, and by regulating the water supply to the heat 
exchanger. Then the cylinder with refrigerant is connected to the oil tank via the pressurereducing valve. Refrigerant R-22 is absorbed by oil. In the course of saturation with the refrigerant, the pressure in the system increases to the set one and the process is monitored using an automatic viscometer, by regular connection of the device to the oil system. The liquid enters the device under a slight excess pressure, which excludes throttling when the mixture enters the viscometer tank. The preset pressure in the vapor chamber of the viscometer is achieved by connecting the device to the cylinder before feeding the mixture into the device. The viscosity is measured several times at regular intervals. Constant viscosity value is defined as viscosity at the state of complete saturation of oil with the refrigerant.

As a result of an experimental study of viscosity properties of oil-refrigerant mixtures in a dynamic mode, data were obtained, on which basis viscosity-temperature dependences for mixtures T-30 - R-22 were designed for refrigerant pressures, respectively: $0-0.35 \mathrm{MPa}, 0$ $-0.45 \mathrm{MPa}$ (fig. 2). The selected pressure range to some extent corresponds to the pressure in the oil supply systems of centrifugal compressors.

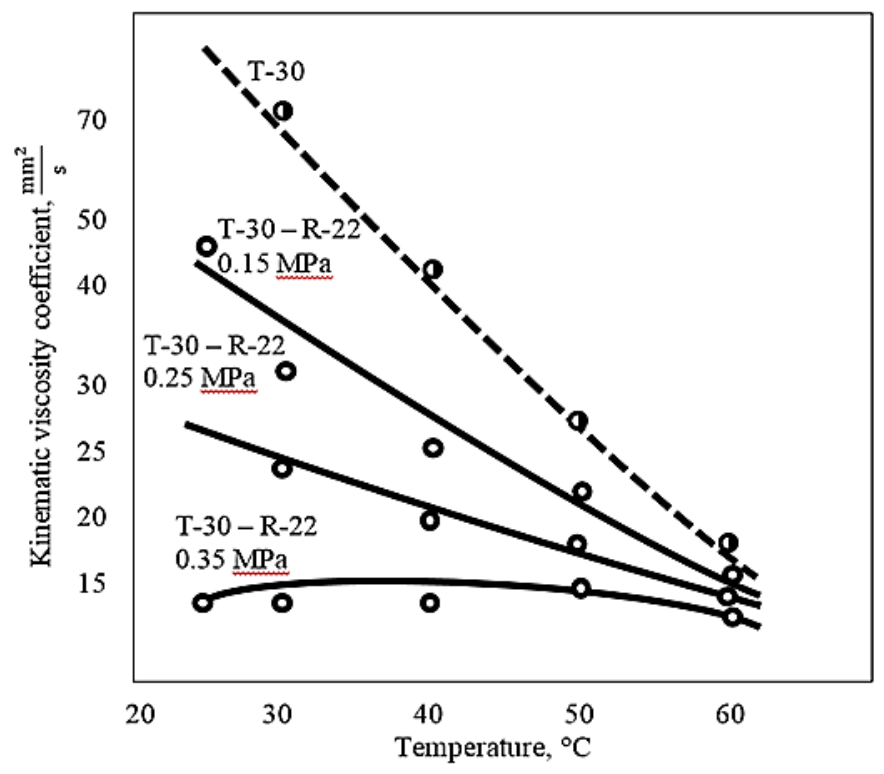

Fig. 2. Viscosity-temperature dependences for mixtures T30-R22.

An attempt to establish the relationship between refrigerant concentration in oil and the length of the lubricating layer of the floating ring of the thrust and radial bearing seal based on the experimental data on viscosity coefficients and mixture concentration (depending on temperature and pressure for an equilibrium state) is represented in [1].

The solution of such a problem for a radial bearing is complicated, firstly, by the fact that friction processes in the loaded and unloaded areas are different; secondly, the throttling of mixture at the entrance into the bearing clearance and foaming in the "boiling zones" changes not only heat intensity, but also viscosity of lubricant, and thirdly, hydrodynamic pressure distribution diagrams undergo changes due to lubricant flow discontinuity (fig. 5).

In this regard, it became necessary to create an experimental device that would allow not only to conduct visual observations of the flow of an oil-refrigerant mixture foaming flow in a narrow channel, but also to record the parameters in the foaming area depending on refrigerant concentration in oil, temperature and pressure that depends on clearance geometry and thermal state of the channel surface. 
Device (fig. 3) consists of an oil tank 1 where oil is in contact with a refrigerant, for example, freon, coming from a cylinder 6 through a pressure reducing valve 7 . Oil pump 2 delivers the mixture through a heat exchanger 3 and a valve 9 with an adjusting washer to the experimental assembly - narrow channel 4. From the channel the mixture is drained through a manifold 6 into the oil tank.

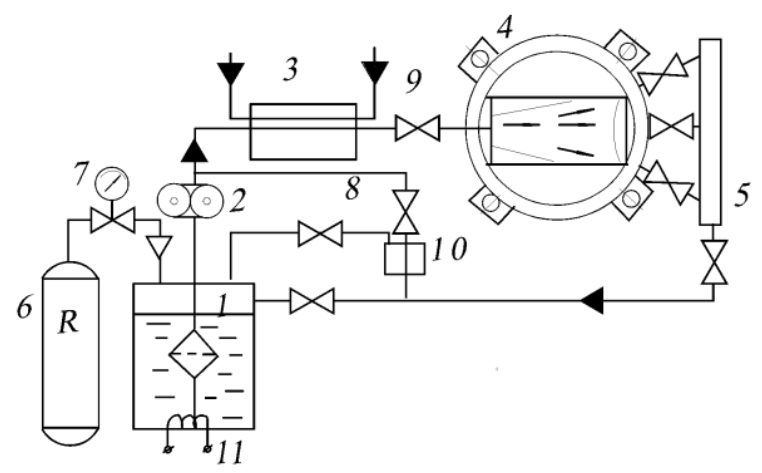

Fig. 3. Scheme of technique for determining viscosity of oil-refrigerant mixture circulating in the lubrication system.

The experimental assembly includes a housing 1 (fig. 4), on which a cover 2 is installed using bolts 3 . A quartz glass disc 4 (diameter $110 \mathrm{~mm}$, thickness $20 \mathrm{~mm}$ ) is located between the housing and the cover. The disc and the housing are separated by a gauge block 6 , which allows the clearance to be set with certain dimensions. Oil and refrigerant mixture arrives to the clearance under pressure through a hole 7 and drained into the collector through a port 8 .

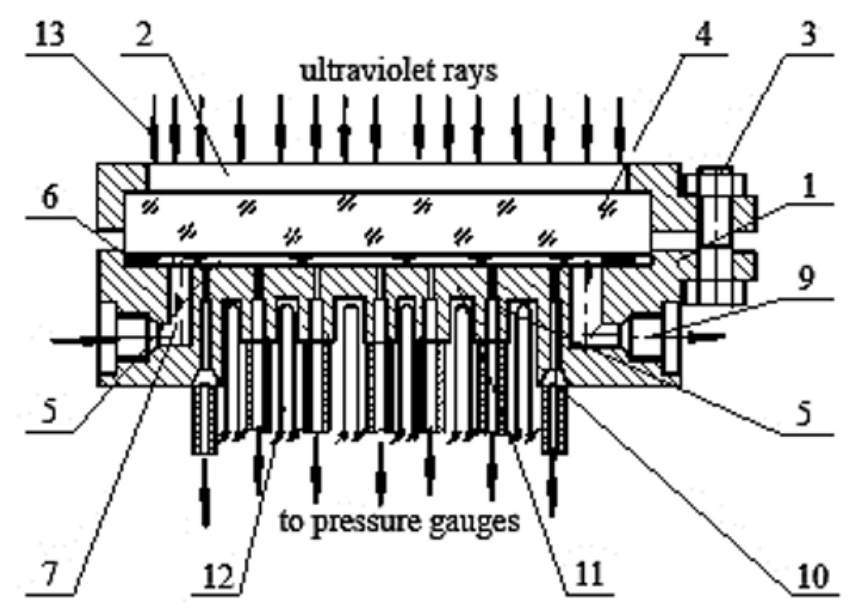

Fig. 4. Experimental assembly.

The design provides for a number of electric heaters and pipes - water coolers, which make it possible to create various thermal fields of the channel surface, which are controlled by 24 copper-constantan thermocouples 11 . The change in the temperature of mixture flow is recorded by an infrared pyrometer, and the pressure drop is recorded by reference pressure gauges of MO type. Visual observations of the lubricant flow in the channel are carried out in ultraviolet rays. 


\section{Results and discussion}

The experience of operating refrigeration screw and compressor-turbine driven units as well as the carried out studies [15] showed that the thermal stress of radial bearings, in most cases, is lower than the calculated one. This is confirmed by the data represented in fig. 2.1 by the axial temperature profiles of a radial bearing operating under the following conditions: Turbine oil T-30 and mixture T-30 - R-22; bearing diameter $0.038 \mathrm{~m}$; sleeve length $\ell=0.035$ $\mathrm{m}$; relative clearance $\delta=0.0065$; shaft rotation frequency $\mathrm{n}=200 \mathrm{rpm}$; specific pressure 0.45 $\mathrm{MPa}$; lubricant pressure at the inlet $0.15 \mathrm{MPa}$; temperature of the lubricant at the bearing entry $40^{\circ} \mathrm{C}$; refrigerant concentration in oil $5 \%$.

A comparative assessment of the dependencies shows that in the axial and circumferential directions the bearing temperature when lubricated with a mixture of T-30 - R-22 is significantly lower than when lubricated with T-30 oil. Moreover, the difference in average temperatures for this case comprised $\Delta \mathrm{T}=10-12{ }^{\circ} \mathrm{C}$. Significant temperature gradients in the axial direction in the bearing loaded area indicate the presence of an additional heat removal at its ends. Moreover, a change in the nature of hydrodynamic pressure distribution, in particular, in the plane passing through the line of action (fig. 5) and the bearing axis, caused by a pressure drop at the ends, makes it possible to say about violation of the lubricant flow continuity and decrease in the bearing capacity.

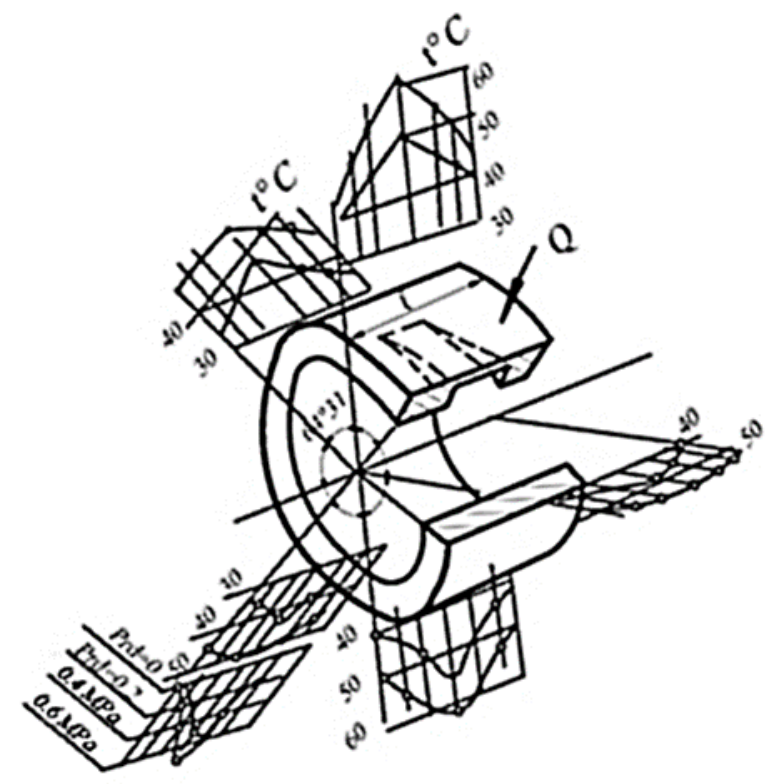

Fig. 5. Hydrodynamic pressure distribution diagrams.

Combination of these phenomena is caused by lubricant foaming in the diffuser zone and at the ends of the bearing where the following conditions are met:

$$
\begin{array}{ll}
P=P_{R} & \frac{d P}{d \varphi}=0 ; \\
P=P_{R} & \frac{d P}{d \ell}=0 ;
\end{array}
$$

where: $\quad P$-hydrodynamic pressure in the lubrication layer, $P_{R}$ - refrigerant vapors saturation pressure, $\varphi$ - circumferential coordinate, $\ell$ - bearing length

, which, according to experts, will reduce the cost of the work done by about $10-15 \%$. 


\section{Conclusion}

Based on the foregoing, it can be concluded that the use of modern methods for calculating bearings of refrigeration units is acceptable if they take into account thermal-physical properties of oil-refrigerant mixtures as well as their behavior in the clearances of friction joints.

The first series of experiments was devoted to the visual study of the oil flow in a slot with variable cross-section. In this case, the presence of air bubbles in the lubricant flow was observed. Bubbles having a diameter of 0.1 to $0.5 \mathrm{~mm}$ had a stable elliptical shape in motion. In the case of presence of bubbles of a larger diameter in lubrication, when moving the slot in the diffuser zone the bubble increased in size, ruptured and lubricant foaming occurred.

Mutual solubility of freons in oil leads to a lubricant viscosity change and has a significant effect on the performance of friction joints in refrigeration units.

\section{References}

1. Kasper Górny Arkadiusz Stachowiak Przemysław Tyczewski Wiesław Zwierzycki. International Journal of Refrigeration. Volume 69, Pages 261-271 (2016)

2. Wang Chuang, Xing Ziwen, Hou Feng, Wu Huagen, Yu Zhiqiang. International Journal of Refrigeration. Volume 90, Pages 1-11 (June 2016)

3. Wu Jianhua Chen Zhenhua Lin Jie Li Jinbo. International Journal of Refrigeration. Volume 94, Pages 24-32 (October 2018)

4. Yanjun Sun, Jian Wang, Xiaopo Wang, Maogang He, Yusheng Hu. International Journal of Refrigeration. Available online 28 October (2020)

5. M. Wasim Akram, Kyriaki Polychronopoulou, Andreas A. Polycarpou. Tribology International Volume. 78, Pages 176-186 (October 2014)

6. M. Wasim Akram, Kyriaki Polychronopoulou, Christopher Seeton, Andreas A.Polycarpou. Wear. Volume 304, Issues 1-2, 15, Pages 191-201 (July 2013)

7. Rui Zhai, Zhao Yang, Biao Feng, Zijian Lv, Wenzhong Zhao, Yubo Chen. Applied Thermal Engineering. Volume 159, 113811 (August 2019)

8. Mitsuhiro Fukuta, Junki Sumiyama, Masaaki Motozawa, Weerachai Chaiworapuek. International Journal of Refrigeration. Volume 119, Pages 131-138 (November 2020)

9. Mathieu Garcia, Benyebka Bou-Saïd, Jérôme Rocchi, Grégory Grau. Tribology International. Volume 65, Pages 363-369 (September 2013)

10. Bachir Bouchehita, Benyebka Bou-Saïda, Mathieu Garcia. Tribology International. Volume 96, Pages 326-348 (April 2016)

11. BinYang, Craig R. Bradshaw, Eckhard A. Groll. International Journal of Refrigeration. Volume 36, Issue 7, Pages 1925-1937 (November 2013)

12. Yajing Lia, Feng Liang, Yu Zhou, Shuiting Ding, Farong Dua Ming Zhou, Jinguang Bi, Yi Cai. Applied Thermal Engineering. Volume 121, Pages 27-38 (July 2017)

13. G.N. Den, Yu.P. Shishkin, A.I. Yakimenko Improving the efficiency of friction units. Collection of scientific works LTIFI, - P. 57 - 61. (1981)

14. Z. Suganami. Friction and lubrication problems. №. 1. - P. 23-30. (1979)

15. Yu.P.Shishkin, E.V.Grigorieva. Improving the efficiency of friction units. Sat. trudov. Dalrybvtuz.. P. 117 - 123 (2010) 